\title{
Apoiando Alinhamento Estratégico em Organizações Usando Contratos Eletrônicos Estendidos com BLA
}

\author{
Lilian Florio, Guilherme B. M. Salles, Marcelo Fantinato \\ Escola de Artes, Ciências e Humanidades - Universidade de São Paulo \\ São Paulo - SP - Brasil \\ \{lilian.fs, guilherme.salles, m.fantinato\}@usp.br
}

\begin{abstract}
Through e-contracts, using partner organizations, it is possible to outsource e-services to be composed into business processes, and set SLA (Service Level Agreement), which does not ensure the strategic alignment between IT departments and business areas of each involved organization. This paper presents an extension of an approach for e-contract establishment including the treatment of BLA (Business Level Agreement), used to align the organization's goals and objectives, so that it is possible to ensure that responsibility activities in IT do not cause negative impacts on the business.
\end{abstract}

Resumo. Por meio de contratos eletrônicos é possivel subcontratar de organizações parceiras serviços eletrônicos, a serem compostos em processos de negócio, e definir SLA (Service Level Agreement), o que não garante o alinhamento estratégico entre as áreas de TI e as áreas de negócio de cada organização envolvida. Este artigo apresenta uma extensão de uma abordagem de estabelecimento de contratos eletrônicos incluindo o tratamento de BLA (Business Level Agreement), usado para alinhar metas e objetivos de uma organização, sendo possível garantir que atividades de responsabilidade de TI não causem impactos negativos para o negócio.

\section{Introdução}

Em busca de ascensão no mercado, organizações procuram outras fornecedoras especializadas nos serviços que desejam contratar com o intuito de ganhar competitividade. Um dos tipos de serviços disponibilizados são serviços web que, por usar a internet e linguagem XML para a comunicação entre aplicações, não exige uma plataforma de hardware ou software para uso. Esse paradigma permite que organizações disponibilizem e/ou contratem esses serviços de outras organizações por meio da web.

Contratos eletrônicos são usados para estabelecer o vínculo entre organizações realizando negócios. Ele é formado pela descrição dos serviços e diversos parâmetros de direitos e deveres. Para firmar um acordo, pode haver uma longa negociação em que organizações envolvidas devem entrar em consenso. Isso faz com que o documento seja alterado diversas vezes, gastando-se muito tempo para seu estabelecimento. Fantinato, Toledo e Gimenes (2010) propõe uma abordagem, chamada PL4BPM, para o estabelecimento de contratos eletrônicos para fornecimento e/ou consumo de serviços web, incluindo processos de negócio e atributos de qualidade de serviço (QoS - Quality of Service), usando linguagens de especificação já existentes. Nesse trabalho, o conjunto de cláusulas de QoS dentro do contrato é chamado de SLA (Service Level Agreement). 
SLA se refere a "Acordo em Nível de Serviço" entre duas organizações, ou seja, define as obrigações das partes envolvidas. Esse conjunto de cláusulas, no contrato, explicita como deve ser a execução do serviço contratado para garantir disponibilidade, tempo de resposta, entre outros parâmetros essenciais para os serviços contratados.

Apesar do apoio da área de Tecnologia da Informação (TI) para implantação de novas tecnologias e melhorias no negócio, muitas organizações estão desacreditadas na TI, pois a TI é distante e não está alinhada com seus objetivos estratégicos, causando muitas vezes gastos desnecessários. A falta de habilidade das organizações em obter retornos satisfatórios dos investimentos em TI deve-se, em parte, à falta de alinhamento entre as estratégias de negócio e as de TI [Laurindo et al. 2001; Brodbeck et al. 2005].

Assim, SLA pode não ser suficiente para garantir o bom funcionamento da troca de serviços eletrônicos. Portanto, é importante buscar uma definição relacionada à SLA, de mais alto nível, para inserir em contratos eletrônicos entre duas organizações e que seja uma forma de relacionar a TI com as áreas de negócio das organizações envolvidas. Hierarquicamente, existe o "Acordo em Nível de Negócio" ou BLA (Business Level Agreement), que pode ser mapeado para um conjunto de SLA e reflete nas relações de negócio entre as organizações envolvidas. BLA é um acordo realizado no nível de negócio que define a forma com que os parceiros devem seguir ao negociar qualquer tipo de serviço eletrônico a ser fornecido ou usado entre eles [Grgic e Rohne 2001].

Nesse cenário, este trabalho trata dos benefícios que o alinhamento estratégico de TI baseado no uso conjunto de BLA com SLA pode trazer para as organizações envolvidas e propõe a implementação de uma extensão na abordagem PL4BPM. Essa abordagem e sua ferramenta - Feature Contract (Feature Modeling based Web Services E-Contracts establishment tool) - têm o objetivo de realizar contratos eletrônicos para serviços web, compostos por três partes, especificadas por linguagens baseadas em XML: serviços eletrônicos, níveis de QoS, e processo de negócio.

Este artigo contém: Seção 2 com panorama sobre alinhamento estratégico; Seção 3 com visão geral sobre a abordagem sendo estendida; Seção 4 com a estratégia de inclusão de BLA na abordagem PL4BPM; Seção 5 com um exemplo de aplicação da estratégia definida; Seção 6 com lições aprendidas; e, Seção 7 com a conclusão.

\section{Alinhamento Estratégico e Trabalhos Relacionados}

Uma atual preocupação das organizações é investir em TI para trazer novas tecnologias que favoreçam seu desenvolvimento, gerando vantagem competitiva. De acordo com Albertin (2001), a TI é uma das principais bases para as organizações realizarem seu planejamento e criarem estratégias voltadas para o futuro.

Luftman et al. (1993) afirmam que o alinhamento estratégico não é um evento isolado, mas um processo de adaptação contínua. Assim, esse processo de exploração da TI de maneira contínua consiste na capacidade de uma organização em implementar tecnologias que a torne diferenciada de seus competidores.

Entretanto, tecnologias desalinhadas com objetivos organizacionais podem ser sinônimos de altos custos e baixa lucratividade. A principal questão é como manter o alinhamento estratégico em TI quando serviços ou partes do processo são terceirizados, 
considerando que para garantir o sucesso da organização contratante é necessário que as organizações contratadas entreguem resultados conforme as diretrizes da cliente.

Nesse contexto, SLA e BLA são temas atuais. Enquanto SLA é um acordo para formalizar serviços a serem contratados e os índices que precisam ser atingidos para o cumprimento do conjunto de compromissos acordados [Sturm et al., 2000], no caso deste estudo para acordar atributos e níveis de QoS, BLA é ainda recente na área de gestão de processos de negócio. Para Pulier e Taylor (2008), BLA é um acordo que estabelece padrões para processos de negócio. Caso uma métrica deixe de ser cumprida, afetará diretamente o desempenho dos resultados da organização.

É possível comparar SLA e BLA usando uma escala de maturidade organizacional, conforme mostra a Figura 1. SLA é descrito por componentes, tem o foco em serviços de TI e é mensurado por meio de critérios técnicos de infraestrutura e aplicações. BLA está no outro extremo e abrange o serviço eletrônico fim-a-fim, deve estar alinhado para medir se as metas do negócio foram atingidas. Entre os dois acordos, há o Business Service Level Agreement (BSLA), que mede operações de processos.

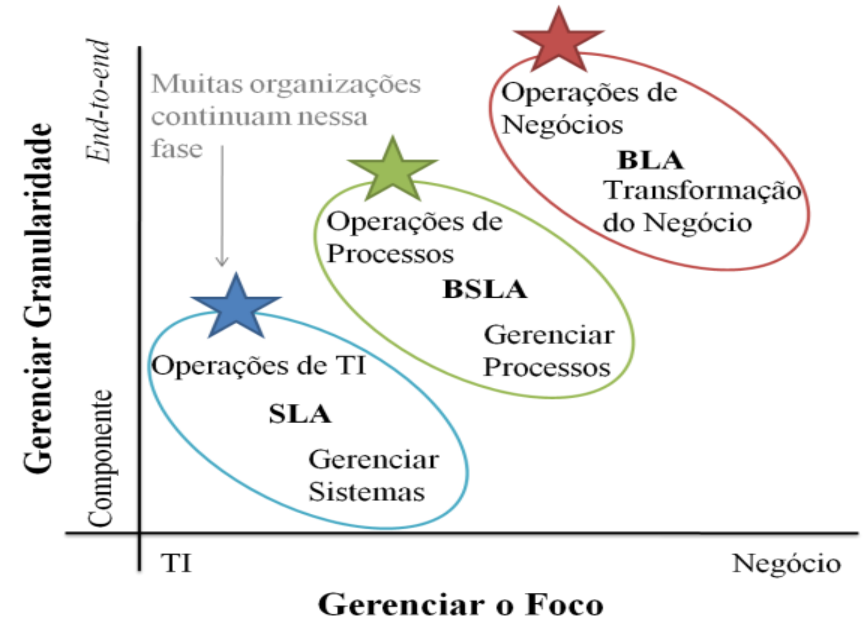

Figura 1. Grau de maturidade da TI dentro de uma organização [Pappe 2008]

Para organizações compartilhando o mesmo processo de negócio composto por serviços eletrônicos, a relação de parceria é importante. Nesse cenário, BLA pode estar associado a etapas de processo de negócio que inclui um conjunto de serviços, cada um por sua vez associado a diferentes SLA; por exemplo com o objetivo de alavancar as vendas e/ou lucros da organização e relacionado a essa métrica pode haver penalidades e incentivos financeiros, o que estreita os laços entre as organizações consumidora e prestadoras de serviços. As organizações necessitam criar parcerias de negócio, sejam clientes ou fornecedores, precisando de um modelo de BLA como complemento ao SLA para garantir que os objetivos de negócio sejam atingidos [Allen et al. 2006].

\section{A Abordagem PL4BPM para o Estabelecimento de Contratos Eletrônicos}

Para duas ou mais organizações realizarem seus negócios usando computação orientada a serviços e, mais especificamente, serviços web, pode ser necessário o estabelecimento de um contrato. Há diversas questões relacionadas a esse tema que não são escopo deste trabalho, tais como: sua validade legal e assinaturas eletrônicas. 
$\mathrm{Na}$ abordagem base desse trabalho [Fantinato, Toledo e Gimenes 2010], o contrato eletrônico é formado por três artefatos responsáveis por especificar: os serviços disponibilizados, o processo de negócio no qual os serviços web estão inseridos; e, os requisitos não funcionais de QoS que influenciam diretamente no processo de negócio. Esses artefatos são descritos nas linguagens WSDL (Web Services Description Language), WS-BPEL (Web Services Business Process Execution Language) e WSAgreement (Web Services Agreement Specification), respectivamente.

Fantinato, Toledo e Gimenes (2010) usam modelos de características para descrever e selecionar serviços e níveis de QoS na estratégia de estabelecimento de contratos eletrônicos baseada em templates e instâncias de contratos. Modelos de características são diagramas hierárquicos que representam de modo simples e intuitivo particularidades relevantes de um contexto. Essas características podem ser obrigatórias, opcionais ou alternativas [Czarnecki, Helsen e Eisenecker 2005].

Além disso, sua abordagem é baseada em conceitos de Linha de Produto (LP) de software, que objetiva a construção sistemática de software baseando-se em uma família de produtos e em reúso de artefatos [Gimenes e Travassos 2002]. Com base em LP, moldes (templates) de contrato eletrônico são mantidos em um alto nível e, sempre que necessário, instâncias de contrato são geradas com base em um determinado molde.

Das três linguagens usadas na elaboração de contratos eletrônicos para serviços web na abordagem PL4BPM, duas delas são já bastante conhecidas e usadas na área de computação orientada a serviços: WSDL e WS-BPEL. WSDL é a linguagem básica usada para a descrição da interface dos serviços eletrônicos a serem disponibilizados e posteriormente acessados na internet seguindo protocolos web; e WS-BPEL é a linguagem usada para a composição de serviços eletrônicos em serviços mais complexos seguindo um determinado protocolo de execução que define a ordem e regras de execução dos serviços eletrônicos na forma de um processo de negócio.

A terceira linguagem usada - WS-Agreement - permite descrever os serviços oferecidos, suas propriedades e as garantias associadas ao serviço, que pode ser um serviço web [Silva 2008]. Nessa linguagem, é possível descrever garantias com multas e bonificações associadas e relacionando com o cumprimento do acordo entre as organizações. Para serviços web, os principais requisitos de QoS são: disponibilidade, acessibilidade, integridade, desempenho, confiabilidade, regulamentação e segurança [Mani e Nagarajan 2002], também chamados de SLA.

\section{Inclusão do BLA na Abordagem PL4BPM}

Esta seção apresenta a estratégia proposta para incluir o tratamento de BLA na abordagem PL4BPM, agregando valor ao contrato entre organizações e buscando alinhamento estratégico entre TI e as áreas de negócio nas organizações participantes.

Embora não haja uniformização para o conceito de BLA nem proposta padrão de como estruturá-lo dentro de um contrato, ele é entendível e direcionado ao negócio, ou seja aos níveis hierárquicos mais altos da organização [Allen et al. 2006; Pulier e Taylor 2008; Pappe 2008]. BLA não chega estar vinculado a processos de negócio no nível mais alto, mas a algo que compõe os processos, como um grupo de atividades. 
Na abordagem PL4BPM, contratos eletrônicos são firmados para acordar os serviços web que serão fornecidos e/ou consumidos. Nesse contexto, para tratar e definir níveis de serviço (SLA), é usada a linguagem WS-Agreement, que possibilita que as organizações participantes monitorem em tempo real e cobrem por níveis de serviço comprados. WS-Agreement por sua vez pode estar relacionada à WSDL para relacionar a quais serviços web uma determinada regra do SLA se aplica.

Originalmente, WS-Agreement permite alinhar expectativas das organizações em relação aos serviços fornecidos. Considerando sua flexibilidade, este trabalho propõe usar sua estrutura para também relacionar acordos de qualidade às atividades de processos de negócio, criando um novo artefato baseado em WS-Agreement, de mais alto nível, que se relacione a artefatos WS-BPEL, em contraponto a artefatos WSDL (situação comum para SLA), para mostrar uma meta a ser atingida e atribuir garantias a ela. Nesse caso, WS-Agreement estaria sendo usado para especificar um BLA.

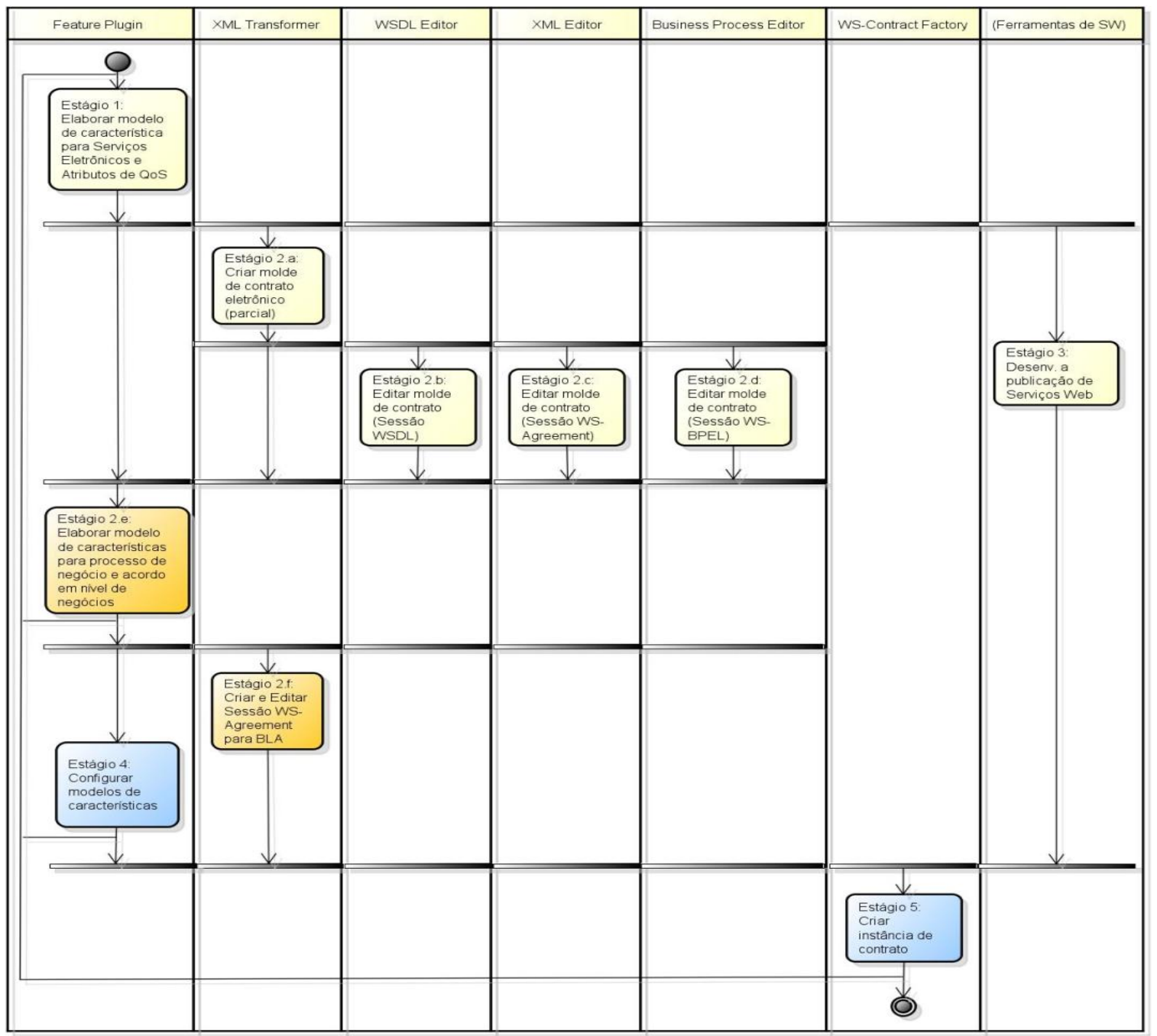

Figura 2. Processo da abordagem PL4BPM estendido com tratamento de BLA

Seguindo o processo original da abordagem PL4BPM, em que os artefatos WSAgreement, para SLA, são gerados a partir dos modelos de características (de uma de suas sub-árvores), é necessário que o processo de negócio em WS-BPEL (criado originalmente a partir de artefatos WSDL que por sua vez são gerados a partir da outra 
sub-árvore dos modelos de características) também seja mapeado para um novo modelo de características. A partir de uma das sub-árvores desse novo modelo, o novo artefato WS-Agreement, para BLA, é gerado. O processo para a criação dos artefatos está descrito na Figura 2 e as atividades propostas neste trabalho estão nos estágios 2.e e 2.f.

Visando a transformação do processo de negócio WS-BPEL em um modelo de características (etapa 2.e da Figura 2), foram desenvolvidos dois meta-modelos, apresentados na Figura 3. O primeiro (Figura 3(a)), estendido do meta-modelo de Fantinato (2007), apresenta o molde de contrato eletrônico, ou seja, contém todas as seções do contrato: WSDL, WS-Agreement, WS-BPEL e WS-Agreement para BLA (o qual faz parte da extensão). O segundo (Figura 3(b), é o meta-modelo para o modelo de características e representa a estrutura padrão do relacionamento entre o processo de negócio e os termos de qualidade de BLA.

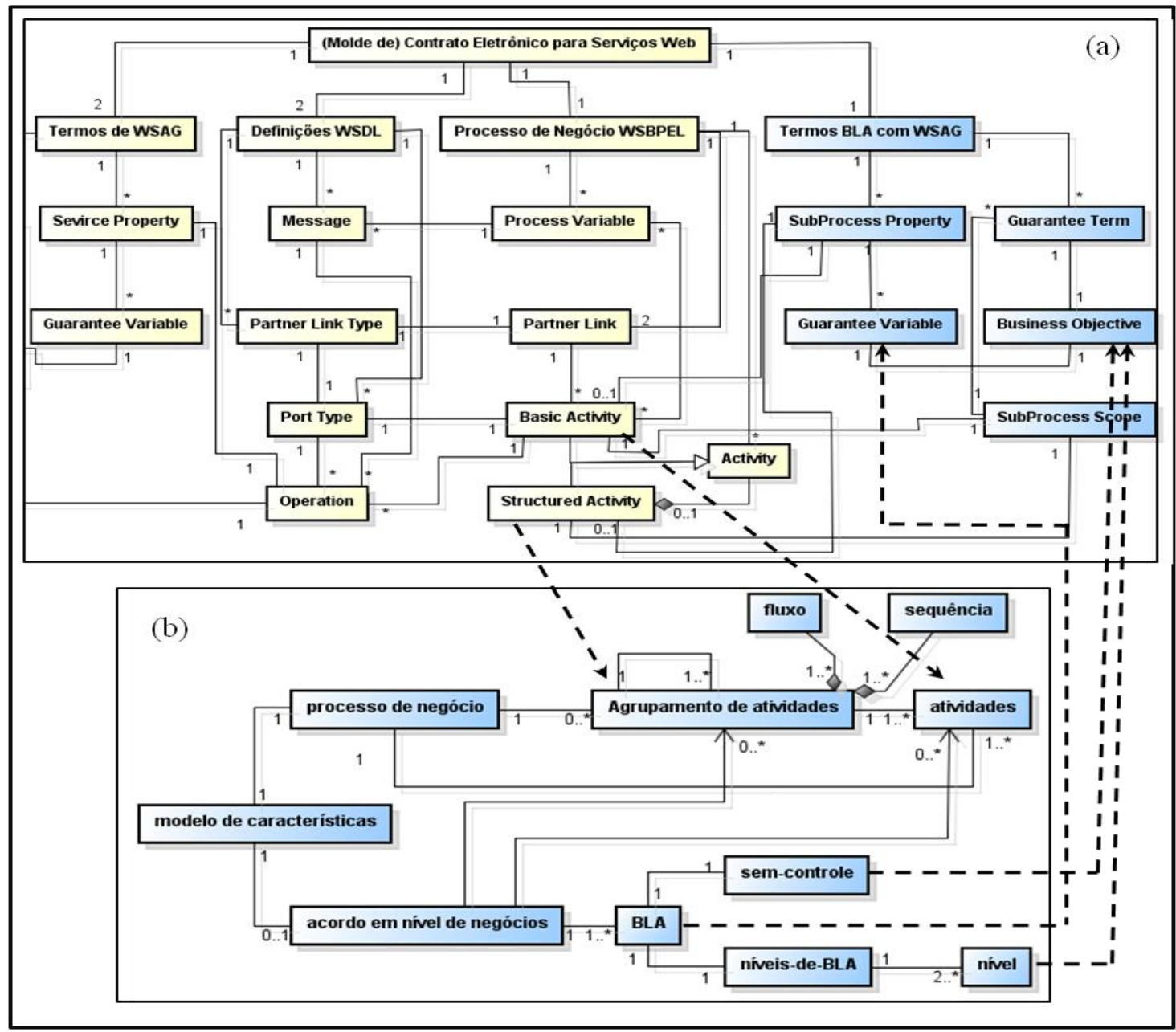

Figura 3. Mapeamento entre elementos dos meta-modelo de contrato eletrônico e meta-modelo de características para processos de negócio e BLA. (a) Metamodelo de contrato eletrônico para serviços web estendido de Fantinato (2007); (b) meta-modelo de características para processo de negócio e BLA.

O meta-modelo de contrato é uma visão em alto nível dos artefatos que compõe o contrato eletrônico e suas respectivas linguagens de especificação, de modo que apenas os elementos mais relevantes estão representados. Apenas a cardinalidade necessariamente relacionada à proposta deste trabalho está apresentada. 
O modelo de características (Figura 3(b)) possui duas sub-árvores obrigatórias: Processo de Negócio e BLA. A primeira contém as sequências e/ou fluxos com a distribuição de atividades dentro do processo de negócio definido pela visão do consumidor. A segunda é composta pelas metas ou sub-metas da organização, que são influenciadas pelos serviços prestados. Assim é possível definir o BLA e determinar qual o nível de entrega que o cliente deseja, de forma associada a atributos e níveis.

Primeiramente, para gerar a sub-árvore do processo de negócio, a partir da seção WS-BPEL do meta-modelo do molde (Figura 3(a)), algumas partes foram associadas para mostrar qual informação estará presente no modelo. As características Basic Activity e Structure Activity são indispensáveis para criar a sub-árvore de processo de negócio. Esses elementos serão do tipo de atividade e subprocessos, como fluxos e sequências. Por não ser o foco principal deste trabalho, a etapa de transformação da seção WS-BPEL para o modelo de características está sendo realizada ainda de forma manual, conforme as regras de relacionamento e cardinalidade entre os meta-modelos.

Uma vez gerada a sub-árvore de processo de negócio, o usuário deve gerar a sub-árvore BLA de acordo com as necessidades da organização ligando com a primeira.

A Tabela 1 contém os tipos de características associados aos atributos de acordo com o que foi definido no meta-modelo do modelo de características. Essas siglas são necessárias para a identificação das características dentro do modelo, informação a ser usada pela ferramenta de suporte FeatureContract, na geração do artefato WSAgreement para BLA, similar ao processo de geração do WS-Agreement para SLA.

Tabela 1. Tipo de característica associado ao atributo do processo de negócio

\begin{tabular}{|l|l|}
\hline Sigla do atributo & Tipo de Característica Associado ao Atributo \\
\hline MC & Modelo de caracteristicas \\
\hline PN & Sub-árvore "processo de negócio" \\
\hline FL & Fluxo de Processo \\
\hline SQ & Sequência do Processo \\
\hline AT & Atividade do Processo \\
\hline
\end{tabular}

Para gerar o artefato WS-Agreement para BLA associado ao processo de negócio em WS-BPEL, usando a ferramenta FeatureContract, muito da estrutura já existente pode ser aproveitada comparando com a geração do artefato WS-Agreement para SLA associado aos serviços eletrônicos em WSDL. As características da sub-árvore BLA do modelo de características, no mapeamento, podem ser: (1) SubProcess Properties/Guarantee Variables (se houver partes do processo associadas a atributos de BLA, então uma variável de garantia será criada para esse atributo); e, (2) Guarantee Terms/Business Objectives (caso a variável de garantia seja associada a um atributo de BLA, um termo de garantia com um objetivo de negócio também será criado contendo o nível de BLA esperado, com os níveis existentes no modelo de características).

\section{Definindo Atributos e Níveis de BLA - Exemplo de Aplicação}

Para verificar a aplicabilidade e analisar benefícios e limitações da extensão proposta, foi realizado um exemplo de aplicação cujos resultados são apresentados nesta seção.

\subsection{Domínio da Aplicação: Operadora de Telecomunicações}

O exemplo abordado trata da integração entre dois sistemas, um de apoio a negócios e um de apoio a operações para organizações operadoras de telecomunicações. Os 
sistemas alvo dentro do contexto abordado são: o sistema de atendimento a clientes (CRM - Customer Relationship Management), um sistema para gerenciar o relacionamento entre organização e cliente como, por exemplo: venda de produto e serviços; criação de contratos; e, registro, consulta e cancelamento de ordens de serviços; e, o sistema de cobranças (COB), que oferece apoio a outros sistemas/organizações para a cobrança de débitos de clientes, como: aplicação, atualização e cancelamento de ações de cobrança; e, aplicação, atualização e cancelamento de reversões de ação de cobrança.

\subsection{Artefatos Produzidos}

A PL4BPM produz um template de contrato eletrônico e várias instâncias de contrato com base nesse template, ambos compostos de três artefatos referentes às seções do contrato: WSDL, WS-Agreement (originalmente apenas para SLA) e WS-BPEL. Na extensão proposta, existe outro artefato, o WS-Agreement para BLA, com o objetivo de relacionar as metas de negócio a atividades do processo ou agrupamento de atividades.

Como etapas do processo já existente da abordagem PLABPM, primeiramente, a ferramenta FeaturePlugin [Czarnecki e Antkiewicz 2004], parte da ferramenta FeatureContract, foi usada para a elaboração de modelos de características. Cada modelo deve conter informações sobre os serviços eletrônicos oferecidos por uma organização e os atributos e níveis de QoS que devem estar relacionados aos serviços. Esse modelo será posteriormente compartilhado entre as organizações para que cada uma possa escolher o que deseja consumir e o nível de QoS desejado. A partir dos modelos de características, dois artefatos WSDL correspondentes às definições dos serviços eletrônicos e dois artefatos WS-Agreement para SLA são produzidos automaticamente pela ferramenta FeatureContract. Cada organização possui um par de seções WSDL e WS-Agreement no template de contrato eletrônico. Em seguida, a partir dos artefatos WSDL, o artefato referente à seção processo de negócio é automaticamente gerada em WS-BPEL. Todas as seções do template do contrato gerados automaticamente devem passar por uma etapa de ajustes e inclusões manuais.

O processo de negócio tem como ator principal o Sistema CRM, porque esta é a organização interessada em subcontratar os serviços da outra. Dessa forma, a cooperação dos serviços pode ocorrer de duas formas:

1. Sistema CRM invoca serviços web oferecidos pelo Sistema COB. Essa ação é representada pelo termo "invoke" junto ao nome da operação;

2. Sistema CRM recebe uma invocação do Sistema COB / Sistema CRM responde à invocação realizada pelo Sistema COB. Esses elementos estão representados no modelo pelos termos "request" e "response", respectivamente.

A principal diferença entre os termos está relacionada ao ator principal. Se o Sistema CRM invoca o COB, o recebimento da resposta do segundo sistema está dentro do escopo de operação do mesmo e, por isso, não é apresentado no processo de negócio.

Passando agora para as etapas da extensão da PL4BPM, escopo deste trabalho, primeiramente é necessário mapear o processo de negócio WS-BPEL gerado para um novo modelo de características. Após completada a transformação de maneira manual, a sub-árvore de BLA pode ser desenvolvida. O principal objetivo dessa fase é, por meio 
de um modelo estruturado hierarquicamente, relacionar objetivos, metas e sub-metas de negócio da organização do Sistema CRM para que a organização provedora do Sistema COB seja uma parceira de negócio e auxilie a obtenção de seus objetivos.

$\mathrm{Na}$ Figura 4 foram associados atributos de BLA a alguns grupos de atividades ou apenas atividades do processo de negócio criado. Para o grupo de atividades que contém Sequencia-acoes-de-AC podem ser atribuídos alguns atributos de BLA como metas-decobrancas, duração-do-sub-processo e/ou dados-atualizados. Cada atributo de BLA contém duas sub-características, sem controle e com níveis, que são excludentes.

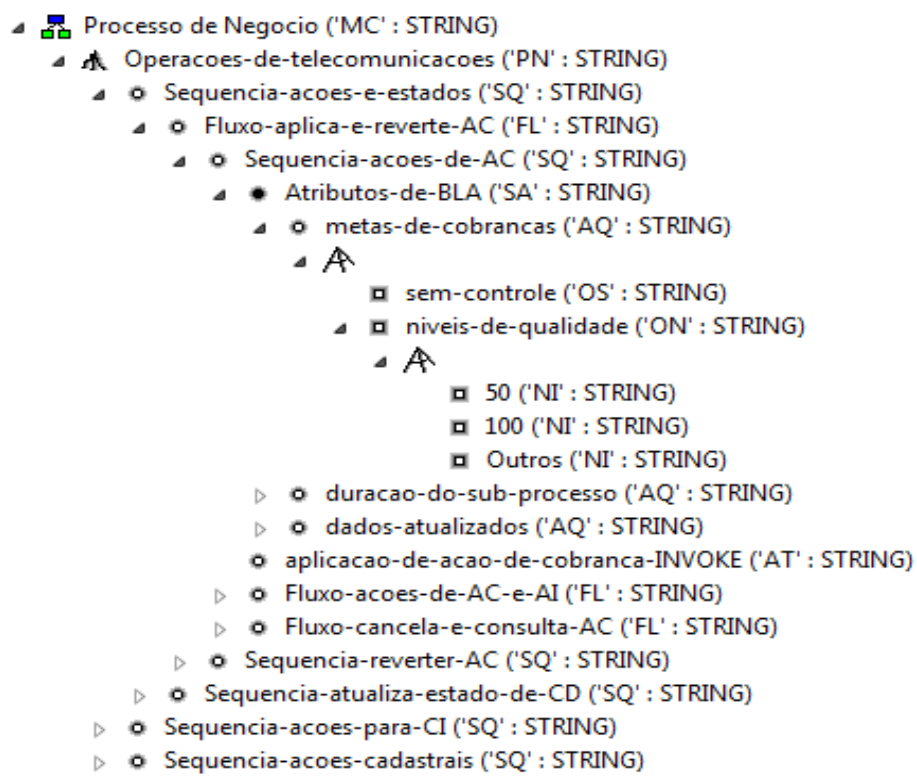

Figura 4. Modelo de características - Processo de Negócio e BLA

A sub-árvore processo de negócio compreende os sub-processos e/ou atividades. Para manter o relacionamento entre esse modelo de características e o arterfato WSBPEL usado como origem para o modelo, os nomes usados para as características são semelhantes à tag name da operação no WS-BPEL que deu origem ao modelo (ex: operação aplicação-de-acao-de-cobranca com o name "aplicacao-de-acao-de-cobrancaINVOKE"). Os atributos estão nomeados conforme as siglas apresentadas na Tabela 1.

A seguir, é criado a quarta seção do contrato eletrônico - o WS-Agreement para BLA, apresentado parcialmente na Figura 5. Esse documento contém referências para agrupamento de atividade ou atividades simples do WS-BPEL que estão associadas a metas de negócio e todas as informações para esclarecer as métricas acordadas.

WS-Agreement para BLA, similarmente para SLA, é composto por duas partes, o SubProcessProperties e o GuaranteeTerms. A primeira parte é composta pelo nome e o tipo de sub-processo: parte do processo, sendo fluxos ou sequências, ou atividade, podendo ser do tipo invoke, request ou response. Na segunda, há um BusinessObjective associado à garantia. Neste há duas possibilidades: criar um objetivo de negócio sem controle ou associar sub-características de opções de atributo para níveis de qualidade. Na Figura 5, pode-se ver o código da seção WS-Agreement para BLA do template de contrato. $\mathrm{O}$ acordo em nível de negócios está associado a parte do processo "Sequenciaacoes-de-AC" e seus BusinessObjective à variável metas-de-cobranca-VAR podem ser sem-controle, de 50 cobranças por dia e assim por diante. 


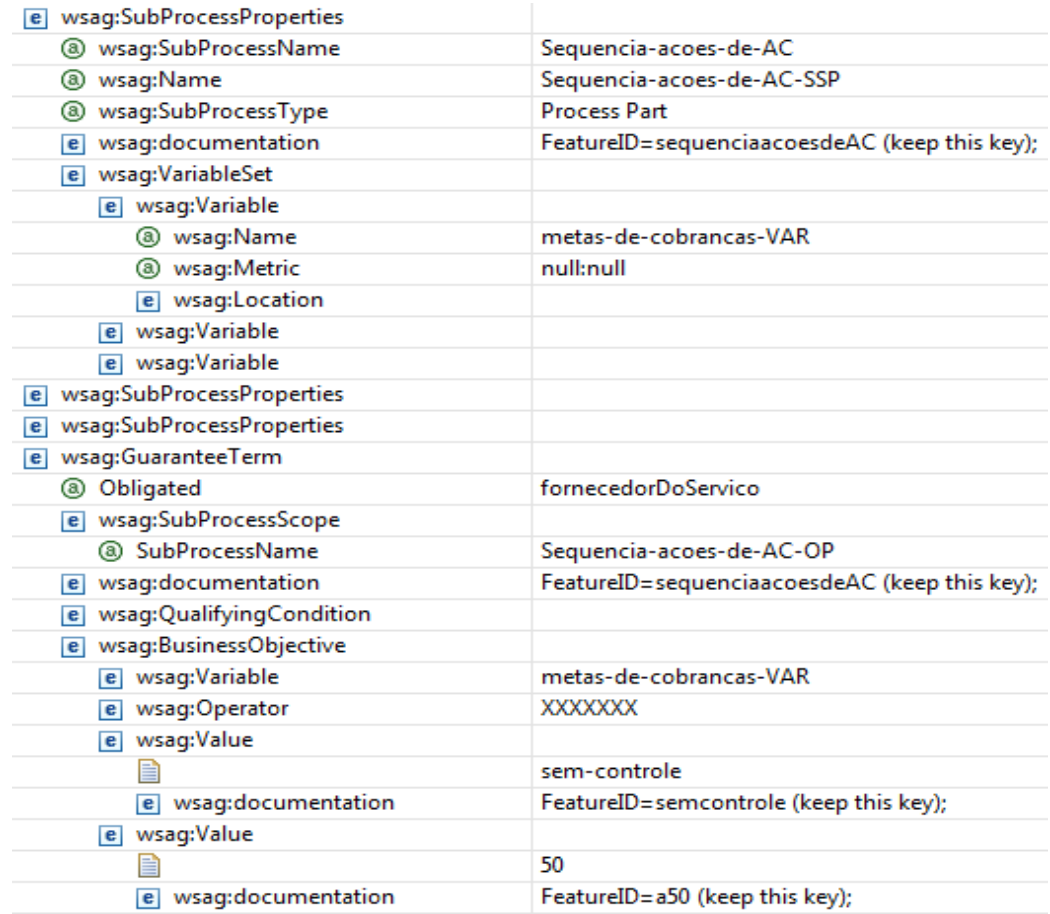

Figura 5. Parte da seção WS-Agreement para BLA

Uma vez gerado completamente o template de contrato com suas quatro seções, é possível iniciar a instanciação de contratos específicos. Para isso, primeiramente devese configurar os modelos de características, incluindo o novo modelo que define possíveis níveis para os atributos de BLA associados a diferentes partes do processo de negócio a ser contratado. Essa etapa deve ser realizada em conjunto pelas organizações porque ambas precisam concordar que é possível garantir a entrega do BLA e que o BLA satisfaz as necessidades da organização contratante. $O$ processo resulta na geração de uma instância de contrato contendo quatro artefatos: WSDL, WS-BPEL, WSAgreement para SLA, e WS-Agrement para BLA.

\section{Lições Aprendidas}

O modelo estrutural proposto para representar BLA apresenta-se robusto por usar WSAgreement, usando suas facilidades como: (i) descrição do objeto e suas propriedades; e, (ii) garantias associadas. Anteriormente a esta proposta, duas organizações usando a abordagem PL4BPM não estariam em sintonia porque, apesar de SLA definidos, não há garantia que a organização cliente está atingindo suas metas de negócio, causando a descrença do negócio na área de TI. Usar o modelo WS-Agreement é factível visto que, de acordo com Allen et al. (2006), BLA é parte da estrutura de linguagem natural de SLA e, dessa forma, conclui-se que há garantias e níveis vinculados ao acordo.

Para gerar os termos de BLA, este trabalho propôs relacioná-los a atividades ou grupos estruturados de atividades de um processo de negócio; necessitando a prévia transcrição do WS-BPEL em modelo de características. As duas grandes vantagens de se usar modelos de características nesse caso, também presentes na estratégia PL4BPM original, são: (i) reúso de artefatos, que facilita e diminui o tempo de criação de novas instâncias de contrato; e, (ii) visualização do processo de negócio de uma maneira simples para realizar as associações com BLA. 
Esta proposta possui também algumas limitações que precisam ser mais bem trabalhadas para que ela esteja considerada completa, como o caso da conversão manual do WS-BPEL para o modelo de características do processo de negócio. Essa etapa automatizada é importante por facilitar o processo de uma forma geral, evitando também possíveis erros operacionais no momento da transcrição. Uma característica importante da abordagem proposta, que pode ser associada tanto a vantagens como a desvantagens, é que ela está associada a uma abordagem maior e mais complexa de estabelecimento de contratos eletrônicos. Se por um lado isso atribui maior valor agregado a todo o conjunto final, por outro lado ela não pode ser usada de forma independente e seu entendimento também pode ser prejudicado.

Não há estudos anteriores que propõem uma forma estruturada para definir e acordar um BLA. Como este acordo é dinâmico e pode ser alterado com maior frequência que uma SLA seria mais difícil e burocrático utilizar o formato de um contrato normal. Sendo assim, este trabalho pode servir de base para outras propostas para TI apoiar o negócio acordando um BLA alinhado com a estratégia de negócio.

\section{Conclusão}

Este trabalho abordou o problema de como duas ou mais organizações podem subcontratar serviços eletrônicos e, por meio de contratos eletrônicos, garantir o alinhamento estratégico da TI. Estendendo a abordagem PL4BPM, esse trabalho apresentou a inclusão de BLA usando modelos de características e conceitos de LP para traçar uma forma sistemática e eficiente de estruturação e reúso de informação.

Com a tendência do mercado em diminuir custos de atividades que não estão relacionadas ao produto final por meio da terceirização, organizações tendem a manter uma área de TI gerencial e enviar as operações a terceiros. Não havendo uma forma estabelecida em contrato para acordar objetivos influenciados pela TI e alinhar os impactos dos serviços dentro do processo de negócio, fica distante garantir o tão almejado alinhamento estratégico entre TI e áreas de negócio. BLA pode ser usado nesse contexto porque é uma forma de incluir em um contrato eletrônico temas que estão sob controle da TI mas afetam indiretamente o sucesso estratégico da organização.

Allen et al. (2006) apontam que o uso de BLA será mais comum entre serviços web porque o ambiente relacionado exige um monitoramento em tempo real, em diversos níveis de abstração. As linguagens apresentadas neste trabalho oferecem essa facilidade. Este trabalho buscou apresentar uma abordagem em que a estratégia de BLA pode ser usada como extensão a uma outra estratégia que usava o gerenciamento de QoS apenas no nível mais baixo, ou seja, SLA. Embora ainda se trate de uma abordagem isolada, trata-se de uma importante contribuição para o contexto estudado.

A principal contribuição da abordagem proposta é reunir diversas fontes que tratam sobre o tema atual BLA, propor e implementar uma forma de estruturar esses termos e como associá-los ao negócio para trazer ganhos as duas organizações, tornando-as parceiras de negócio e garantindo alinhamento estratégico.

\section{Agradecimentos}

Este trabalho foi parcialmente financiado pela Fapesp (Fundação de Amparo à Pesquisa do Estado de São Paulo). 


\section{Referências}

Albertin, A. L. (2001). Administração de informática: funções e fatores críticos de sucesso, Ed. Atlas, terceira edição.

Allen, P. et al. (2006). Service Orientation: Winning Strategies and Best Practices, Cambridge University Press.

Brodbeck, A. F. et al. (2005). Práticas de alinhamento estratégico promovidas em organizações do estado do Rio Grande do Sul, Em Anais do XXXV Encontro da $A N P A D$, páginas 1-16.

Czarnecki, K. e Antkiewicz, M. (2004). Mapping features to models: A template approach based on superimposed variants, In Proc. of the 4th Int. Conf. on Generative Programming and Component Engineering, pages 422-437, Springer.

Czarnecki, K., Helsen, S., e Eisenecker, U. W. (2005). Staged configuration through specialization and multilevel configuration of feature models, In Software Process: Improvement and Practice 10(2), pages 143-169.

Fantinato, M. (2007). Uma Abordagem Baseada em Características para o Estabelecimento de Contratos Eletrônicos para Serviços Web, Tese de Doutorado, Universidade Estadual de Campinas.

Fantinato, M., Toledo, M. B. F., e Gimenes, I. M. S. (2010) "Product Line in the Business Process Management Domain". In: Applied Software Product Line Engineering, Edited by Kyo C. Kang, Vijayan Sugumaran and Sooyong Park, Auerbach Publications, USA, p. 497-530.

Gimenes, I. M. S. e Travassos, G. H. (2002) "O Enfoque de Linha de Produto para Desenvolvimento de Software", Em: XXI Jornadas de Atualização em Informática, SBC, p. 01-31.

Grgic, I. e Rohne, M. (2001). Agreements in IP-based networks, In Telektronikk 2/3.2001, pages 186-212.

Laurindo, F. J. B. et al. (2001). O papel da tecnologia da informação (TI) na estratégia das organizações, In Gestão \& Produção 8(2), pages 160-179.

Luftman, J. N. et al. (1993). Transforming the enterprise: the alignment of business and information technology strategies, IBM System Journal, vol. 32, n. 1.

Mani, A. e Nagarajan, A. (2002) "Understanding Quality of Service for Web Services", http://www.ibm.com/developerworks/webservices/library/ws-quality/

Pappe, S. (2008). Making SOA operational: Service management enabling SOA, In Proc. of the 1st Int. SOA Symposium.

Pulier, E. e Taylor, H. (2008) Compreendendo SOA Corporativa, Ciência Moderna.

Silva, M. F. (2008), Uma abordagem para monitoramento de contratos eletrônicos baseada em aspectos, Dissertação de Mestrado, Universidade Estadual de Maringá.

Sturm, R. et al. (2000). Foundations of Service Level Management, Sams, p. 288. 